\title{
Expression of hepatocyte markers in mass-forming peripheral and periductal-infiltrating hilar intrahepatic cholangiocarcinomas
}

\author{
HIROYA IIDA $^{1,2^{*}}$, MASAKI HATA ${ }^{1 *}$, AYAKO KAKUNO $^{3}$, HIROSHI HIRANO $^{4}$, \\ KOJI YAMANEGI $^{1}$, NAOKO YAMADA ${ }^{1}$, HIDEKI OHYAMA ${ }^{1}$, NOBUYUKI TERADA ${ }^{1}$, \\ CHIAKI YASUI $^{2}$, NAOKI YAMANAKA ${ }^{2}$ and KEIJI NAKASHO ${ }^{1}$ \\ ${ }^{1}$ Department of Pathology, Hyogo College of Medicine, Nishinomiya, Hyogo 663-8501; \\ Departments of ${ }^{2}$ Surgery and ${ }^{3}$ Pathology, Meiwa General Hospital, Nishinomiya, Hyogo 663-8186; \\ ${ }^{4}$ Department of Pathology, Nippon Steel Hirohata Hospital, Himeji, Hyogo 671-1122, Japan
}

Received April 4, 2011; Accepted August 17, 2011

DOI: $10.3892 / \mathrm{ol} .2011 .405$

\begin{abstract}
In this study, the expression of hepatocyte markers, including $\alpha$-fetoprotein (AFP), HepPar-1 antigen and arginase-1, was examined immunohistochemically in 14 mass-forming peripheral intrahepatic cholangiocarcinomas (ICCs) that arose from the peripheral portion of the biliary tree, and in 14 periductal-infiltrating hilar ICCs that arose from intrahepatic large bile ducts. Only $2(14.3 \%)$ of the 14 hilar ICCs and $2(14.3 \%)$ of the 14 peripheral ICCs expressed AFP or HepPar-1 antigen. Conversely, arginase-1 was expressed in $8(57.1 \%)$ and $11(78.6 \%)$ of the hilar and peripheral ICCs, respectively, and $4(28.6 \%)$ hilar ICCs and $7(50 \%)$ peripheral ICCs expressed arginase-1 in more than $10 \%$ of the cancer cells. The expression of arginase- 1 did not differ between peripheral ICCs showing major histology of poorly differentiated adenocarcinoma and those showing other major histologies, including well- or moderately differentiated tubular adenocarcinoma or papillary adenocarcinoma. Results of the present study showed that common hepatocyte markers, including AFP and HepPar-1 antigen, are rarely but definitely expressed in hilar and peripheral ICCs, and that a third hepatocyte marker, arginase-1, is expressed at a high rate in both hilar and peripheral ICCs, irrespective of their histology. These results indicate that care should be taken when using arginase-1 as a hepatocyte marker for distinguishing between a poorly differentiated hepatocellular carcinoma and a mass-forming peripheral ICC showing the histology of poorly differentiated adenocarcinoma.
\end{abstract}

Correspondence to: Dr Masaki Hata, Department of Pathology, Hyogo College of Medicine, Nishinomiya, Hyogo 663-8501, Japan E-mail: hata@hyo-med.ac.jp

*Contributed equally

Key words: $\alpha$-fetoprotein,HepPar-1, arginase-1, cholangiocarcinoma, immunohistochemistry

\section{Introduction}

Intrahepatic cholangiocarcinomas (ICCs) arise from the epithelial cells lining the intrahepatic biliary tree. Macroscopically, ICCs are divided into three types; the mass-forming (MF), the periductal-infiltrating (PI) and the intraductal growth (IG) types (1-3). MF-type ICCs form masses in the hepatic parenchyma, while the PI-type grow along bile ducts and the IG-type demonstrate intraductal polypoid or papillary growth.

ICCs are further classified into hilar and peripheral ICCs. Hilar ICCs evolve from the lining cholangiocytes of the large collecting bile ducts in the hilum or the peribiliary glands around them, and their histological characteristics are similar to those of extrahepatic cholangiocarcinomas (4). On the other hand, peripheral ICCs develop from the lining epithelial cells of small collecting bile ducts, interlobular bile ducts, bile ductules or canals of Hering $(3,4)$. Peripheral ICCs arising from interlobular bile ducts, bile ductules or canals of Hering are usually MF-type ICCs, whereas ICCs arising from the large collecting bile ducts are MF-, PI- or IG-type $(3,4)$.

Hepatic progenitor cells are thought to be capable of differentiating into hepatocytes and cholangiocytes, which reside in the most peripheral branches of the biliary tree; i.e., the bile ductules and canals of Hering (5). Therefore, MF-type peripheral ICCs, which arise from bile ductules and canals of Hering, may express hepatocyte markers. In addition, cholangiocytes have been shown to be capable of transforming into hepatocytes, suggesting that hepatocyte markers are expressed by certain hilar and peripheral ICCs (6). Various studies reported the rare expression of hepatocyte markers such as $\alpha$-fetoprotein (AFP) or HepPar-1 antigen in ICCs (7-10). Furthermore, D'Errico et al (11) reported that 4 of 6 peripheral ICCs expressed albumin mRNA. Therefore, in the present study, the expression of hepatocyte markers in 14 MF-type peripheral and 14 PI-type hilar ICCs was examined. Arginase-1 was used as a hepatocyte marker in addition to AFP and HepPar-1 antigen, since arginase-1 has been reported to be a more sensitive hepatocyte marker than HepPar-1 (12). 
Table I. Macroscopic type, major histology, PAS staining and immunohistochemical staining of the hilar and peripheral intrahepatic cholangiocarcinomas.

A, Hilar intrahepatic cholangiocarcinoma.

\begin{tabular}{ccllllllll}
\hline No. & $\begin{array}{c}\text { Macroscopic } \\
\text { type }\end{array}$ & $\begin{array}{c}\text { Major } \\
\text { histology }\end{array}$ & PAS & CK-7 & CK-19 & AFP & HepPar-1 & Arginase-1 & N-CAM \\
\hline 1 & PI & Pap & $(+)$ & $5+$ & $5+$ & - & - & $3+$ & - \\
2 & PI & Por & $(+)$ Focal & $5+$ & $5+$ & - & - & - & - \\
3 & PI & Tub1 & $(+)$ & $5+$ & $5+$ & - & - & - & - \\
4 & PI & Tub1 & $(+)$ & $5+$ & $5+$ & - & - & - & - \\
5 & PI & Tub1 & $(+)$ & $5+$ & $5+$ & - & - & $1+$ & - \\
6 & PI & Tub1 & $(+)$ & $5+$ & $5+$ & - & - & $1+$ & - \\
7 & PI & Tub1 & $(+)$ & $5+$ & $5+$ & - & $2+$ & - & - \\
8 & PI & Tub1 & $(+)$ & $5+$ & $5+$ & - & - & $1+$ & - \\
9 & PI & Tub1 & $(+)$ Focal & $4+$ & $4+$ & - & - & $1+$ & - \\
10 & PI & Tub1 & $(+)$ & $5+$ & $5+$ & - & - & - & - \\
11 & PI & Tub1 & $(+)$ & $5+$ & $5+$ & - & - & $3+$ & - \\
12 & PI & Tub2 & $(+)$ & $5+$ & $5+$ & - & $4+$ & $3+$ & - \\
13 & PI & Tub2 & $(+)$ & $5+$ & $5+$ & - & - & $3+$ & $1+$ \\
14 & PI & Tub2 $>$ Por & $(+)$ & $5+$ & $5+$ & - & - & - & - \\
\hline
\end{tabular}

B, Peripheral intrahepatic cholangiocarcinoma.

\begin{tabular}{rlllllllll}
\hline $\begin{array}{c}\text { No. } \\
\text { Macroscopic } \\
\text { type }\end{array}$ & $\begin{array}{c}\text { Major } \\
\text { histology }\end{array}$ & PAS & CK-7 & CK-19 & AFP & HepPar-1 & Arginase-1 & N-CAM \\
\hline 1 & MF & Pap > Tub2 & $(+)$ & $4+$ & $4+$ & $3+$ & $3+$ & $2+$ & - \\
2 & MF & Por & $(+)$ & $5+$ & $3+$ & - & - & - & - \\
3 & MF & Por & $(+)$ Focal & $5+$ & $5+$ & - & - & $3+$ & - \\
4 & MF & Por & $(+)$ & $5+$ & $5+$ & - & - & $3+$ & - \\
5 & MF & Por & $(+)$ & $5+$ & $5+$ & - & - & $3+$ & $1+$ \\
6 & MF & Por & $(+)$ & $2+$ & $4+$ & - & - & $1+$ & - \\
7 & MF & Por & $(+)$ & $5+$ & $5+$ & - & - & - & - \\
8 & MF & Por $>$ Tub2 & $(+)$ Focal & $5+$ & $5+$ & - & - & $2+$ & $5+$ \\
9 & MF & Tub1 & $(+)$ & $5+$ & $4+$ & - & - & $3+$ & - \\
10 & MF & Tub1 & $(+)$ Focal & $4+$ & $5+$ & - & - & $4+$ & - \\
11 & MF & Tub2 $>$ Tub1 & $(+)$ & $5+$ & $4+$ & - & - & $3+$ & - \\
12 & MF & Tub2 & $(+)$ Focal & $5+$ & $5+$ & $2+$ & - & $3+$ & $4+$ \\
13 & MF & Tub2 & $(+)$ Focal & $5+$ & $3+$ & - & - & - & - \\
14 & MF & Tub2 & $(+)$ & $5+$ & $5+$ & - & - & $1+$ & - \\
\hline
\end{tabular}

PI, periductal-infiltrating type; MF, mass-forming type; Pap, Papillary adenocarcinoma; Tub1, well-differentiated tubular adenocarcinoma; Tub2, moderately differentiated tubular adenocarcinoma; Por, poorly differentiated adenocarcinoma. Staining grade: - , positive cells (p) $<1 \%$; $1+, 1 \% \leq \mathrm{p}<5 \% ; 2+, 5 \% \leq \mathrm{p}<10 \% ; 3+, 10 \% \leq \mathrm{p}<40 \% ; 4+, 40 \% \leq \mathrm{p}<70 \%, 5+, \mathrm{p} \geq 70 \%$.

\section{Materials and methods}

Subjects. Specimens of 14 hilar and 14 peripheral ICCs were used for this study. The specimens were obtained from liver tumors resected at Meiwa General Hospital, Nippon Steel Hirohata Hospital, and the hospital attached to Hyogo College of Medicine, Japan, between 1988 and 2010. Written consent was obtained from each patient prior to surgery, and anonymous usage of tissue samples for pathological studies was permitted. The number of male and female patients with hilar ICCs and peripheral ICCs were 5 and 9 , and 10 and 4, respectively.

Samples. The surgically obtained tumors were fixed in $10 \% 0.01 \mathrm{M}$ phosphate-buffered formalin $(\mathrm{pH}$ 7.4) and cut through the largest area; several samples, including those with the largest area, were prepared and embedded in paraffin. Sections $(5 \mu \mathrm{m})$ of these samples were used for H\&E staining, 
periodic acid Schiff reaction (PAS) staining and immunohistochemical analysis.

Immunohistochemistry. The sources of antibodies and their dilutions were as follows: anti-human HepPar-1 antigen mouse monoclonal antibody (OHC1E5) (25-fold dilution; Dako Japan, Tokyo, Japan), anti-human cytokeratin (CK)-7 mouse monoclonal antibody (OV-TL12/30) (100-fold dilution; Dako Japan), anti-human CK-19 mouse monoclonal antibody (RCK108) (100-fold dilution; Dako Japan), anti-human AFP rabbit polyclonal antibody (100-fold dilution; Dako Japan), anti-human neural cell adhesion molecule (N-CAM) mouse monoclonal antibody (1B6) (pre-diluted; Nichirei Bioscience, Tokyo, Japan) and anti-human arginase-1 rabbit polyclonal antibody (500-fold dilution; Sigma-Aldrich Japan, Tokyo, Japan). The antibodies were diluted with $0.01 \mathrm{M}$ phosphate-buffered saline (PBS) $(\mathrm{pH} 7.4)$ containing $1 \%$ bovine serum albumin (BSA).

The antigen retrieval procedure for the immunohistochemical analysis was: autoclave treatment at $121^{\circ} \mathrm{C}$ for $5 \mathrm{~min}$ in a target retrieval solution (pH 9.0) (Dako Japan) for CK-19, autoclave treatment at $121^{\circ} \mathrm{C}$ for $5 \mathrm{~min}$ in a target retrieval solution (Dako Japan) for HepPar-1 antigen, CK-7, AFP and N-CAM; boiling in a citrate buffer ( $\mathrm{pH}$ 6.0) (Mitsubishi Chemical Medicine Corporation, Tokyo, Japan) at $85-90^{\circ} \mathrm{C}$ for 3 min and cooling at room temperature for arginase- 1 .

To block the internal peroxidase activity and non-specific binding of the primary antibodies, sections were treated with $0.35 \%$ hydrogen peroxide in methanol at room temperature for 15 min and with PBS containing $1 \%$ BSA and $0.1 \%$ Tween-20 at room temperature for $30 \mathrm{~min}$, respectively. Immunohistochemical staining was carried out using an Envision ${ }^{\mathrm{TM}}+$ dual link system (Dako Japan) with a 3,3'-diaminobenzidine (DAB) solution (Nichirei Bioscience). Immunostaining was graded according to the proportion of positive cells $(p)$ : ,$- \mathrm{p}<1 \% ; 1+, 1 \% \leq \mathrm{p}<5 \% ; 2+, 5 \% \leq \mathrm{p}<10 \% ; 3+, 10 \% \leq \mathrm{p}<40 \%$; $4+, 40 \% \leq \mathrm{p}<70 \% ; 5+, \mathrm{p} \geq 70 \%$.

Double immunostaining of $\mathrm{CK}-7$ and HepPar-1 antigen. Sections were first immunostained for CK-7 as described above. These sections were then treated in an autoclave at $121^{\circ} \mathrm{C}$ for $5 \mathrm{~min}$ in a target retrieval solution (Dako Japan) for antigen retrieval and denaturation of the attached antibody and secondary antibody-conjugated horseradish peroxidase, and treated with PBS containing 1\% BSA and 0.1\% Tween-20 at room temperature for 30 min to block any non-specific binding of the anti-HepPar-1 antigen antibody. The sections were then divided into two groups; one group was incubated with the anti-HepPar-1 antigen antibody, whereas the other group was not. The antibody bound to the HepPer-1 antigen was detected using a Histofine ${ }^{\circledR}$ Simple Stain AP (multi) kit (Nichirei Bioscience) with alkaline phosphatase- and secondary antibody-conjugated polymer, and a new fuchsin substrate solution (Nichirei Bioscience) to which levamisole (Dako Japan) was added to block the internal alkaline peroxidase activity.

Statistical analysis. The immunohistochemical staining was analyzed by the Chi-square test using the StatMate III software program for Windows (ATMS, Tokyo, Japan). $\mathrm{P}<0.05$ was considered to be statistically significant.
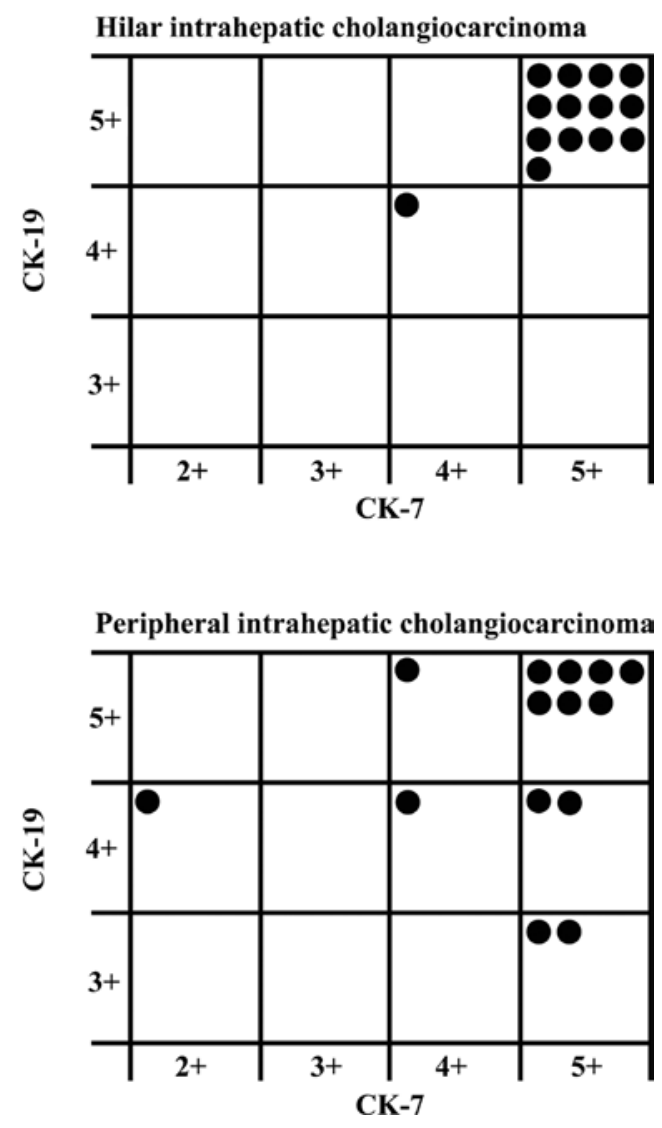

Figure 1. Immunostaining grades of CK-19 and CK-7 in each of the intrahepatic cholangiocarcinomas. The immunostaining grades were: $2+, 5 \% \leq \mathrm{p}$ (positive cells) $<10 \% ; 3+, 10 \% \leq \mathrm{p}<40 \% ; 4+, 40 \% \leq \mathrm{p}<70 \% ; 5+, \mathrm{p} \geq 70 \%$.

\section{Results}

Table I shows the macroscopic type and major histology of the 14 hilar and 14 peripheral ICCs examined in the study, and the results of the PAS and immunohistochemical staining of these ICCs. All hilar ICCs were PI-type, growing along the intrahepatic large bile ducts, and all peripheral ICCs were MF-type. The histological classification was performed according to Nakajima et al (13). The major histology of ICCs was wellor moderately differentiated tubular adenocarcinoma, poorly differentiated adenocarcinoma or papillary adenocarcinoma. Among the 14 hilar ICCs, only one demonstrated the major histology of poorly differentiated adenocarinoma, while among the 14 peripheral ICCs, 7 demonstrated poorly differentiated adenocarcinoma. PAS staining revealed that all hilar and peripheral ICCs produced mucus, but that less mucus was produced by peripheral ICCs than by hilar ICCs.

Fig. 1 shows the grade of immunostaining for CK-19 and CK-7 in each hilar or peripheral ICC. Grade 5 immunostaining for CK-19 and CK-7 was observed in 13 (92.9\%) of the 14 hilar ICCs. Conversely, only 7 (50\%) of the 14 peripheral ICCs revealed grade 5 immunostaining for both CK-19 and CK-7.

Rare hilar and peripheral ICCs expressed AFP or HepPar-1 antigen (Table II, Fig. 2A-C). None of the hilar ICCs revealed positive immunostaining for AFP, and 2 of the hilar ICCs revealed positive immunostaining for HepPar-1 antigen. Positive immunostaining for AFP and HepPar-1 antigen was found in 2 and 1 peripheral ICCs, respectively. AFP or 
Table II. Expression of AFP or HepPar-1 antigen in 14 hilar and 14 peripheral intrahepatic cholangiocarcinomas (ICCs).

\begin{tabular}{lccllc}
\hline \multirow{2}{*}{ Marker } & \multicolumn{2}{c}{ Hilar ICCs } & & \multicolumn{2}{c}{ Peripheral ICCs } \\
\cline { 2 - 3 } \cline { 6 - 7 } & No. $(\%)$ & $\begin{array}{c}\text { Each } \\
\text { grade }\end{array}$ & & No. $(\%)$ & $\begin{array}{c}\text { Each } \\
\text { grade }\end{array}$ \\
\hline AFP & $0(0)$ & - & & $2(14.3)$ & $3+, 2+$ \\
HepPar-1 & $2(14.3)$ & $4+, 2+$ & & $1(7.1)$ & $3+$ \\
AFP or HepPar-1 & $2(14.3)$ & $4+, 2+$ & & $2(14.3)$ & $3+, 2+$ \\
\hline
\end{tabular}

Immunostaining grades were: $2+, 5 \% \leq \mathrm{p}$ (positive cells) $<10 \% ; 3+$, $10 \% \leq \mathrm{p}<40 \% ; 4+, 40 \% \leq \mathrm{p}<70 \%$.

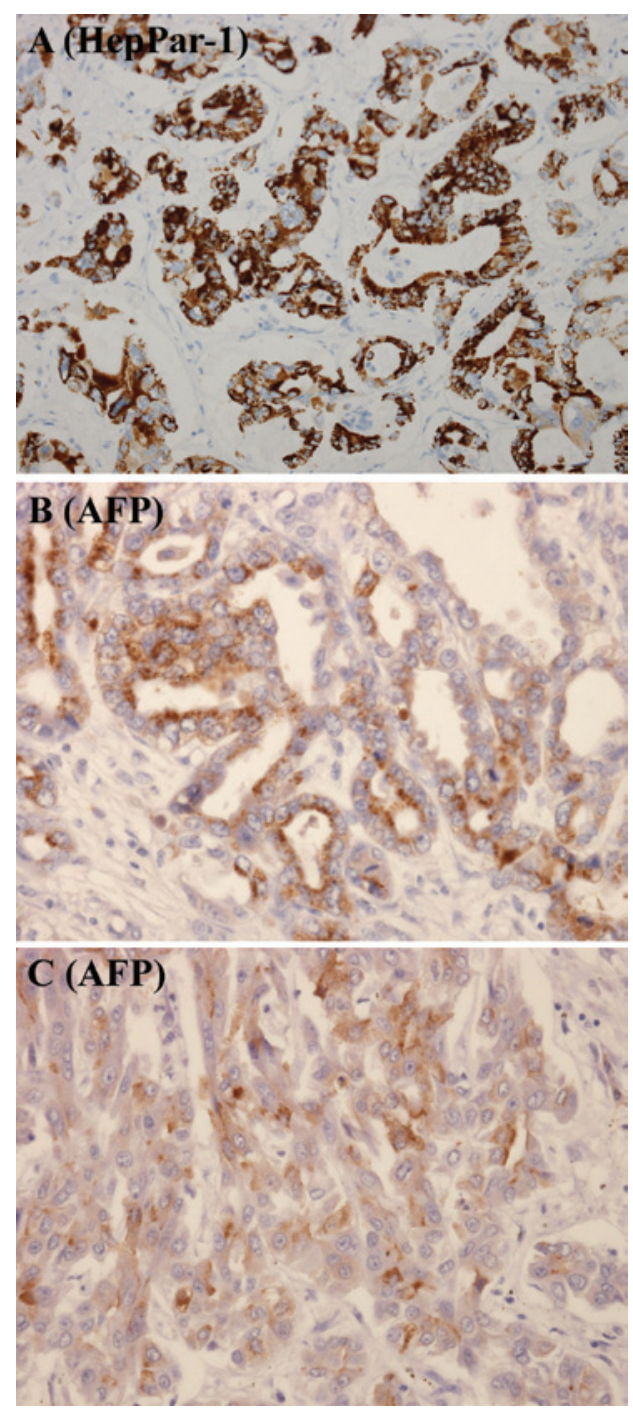

Figure 2. Immunostaining for HepPar-1 antigen and AFP. (A) Immunostaining for HepPar-1 antigen in an area of moderately differentiated tubular adenocarcinoma. (B and C) Immunostaining for AFP in areas of welldifferentiated tubular adenocarcinoma and poorly differentiated adenocarcinoma, respectively. Original magnifications: (A), x200; (B and C), x400.

HepPar-1 antigen was expressed in $2(14.3 \%)$ and $2(14.3 \%)$ of the hilar and peripheral ICCs, respectively. Fig. 3 shows the co-expression of CK-7 and HepPar-1 antigen in cancer cells.

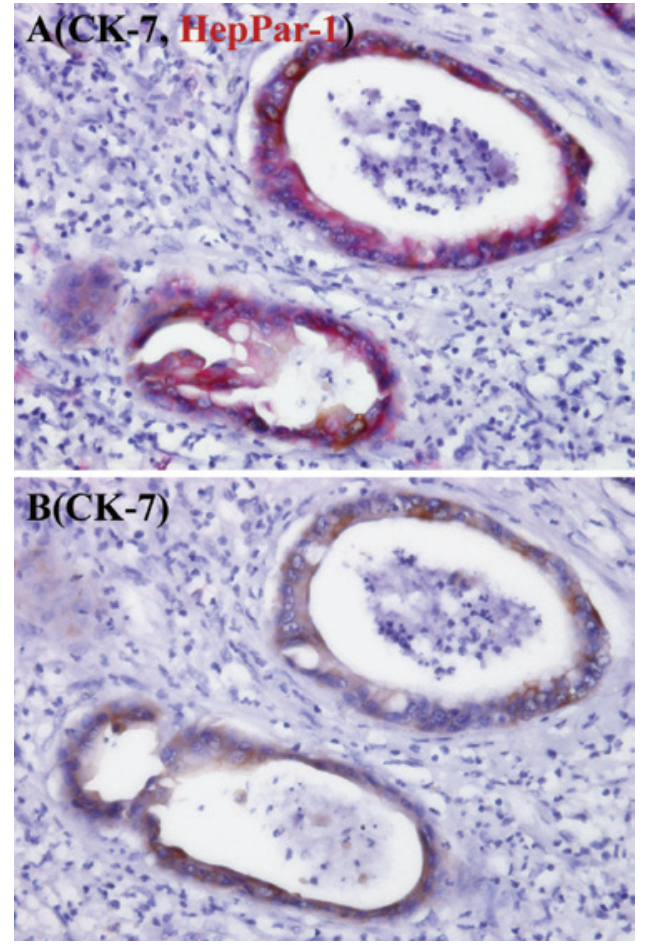

Figure 3. Double immunostaining for CK-7 and HepPar-1 antigen. (A) Double staining of CK-7 (brown) and HepPar-1 antigen (red). (B) Control staining in which the addition of the secondary antibody (anti-HepPar-1 antigen antibody) was omitted. Original magnification, $\mathrm{x} 400$.

Fig. 4 shows the immunostaining grades for arginase-1 in the hilar and peripheral ICCs, and Fig. 5 shows the immunostaining for arginase-1. Immunostaining for arginase-1 at grades 1, 2, 3 or 4 was found in $8(57.1 \%)$ of the hilar ICCs and $11(78.6 \%)$ of the peripheral ICCs. Immunostaining for arginase- 1 at grades 3 or 4 was found in 4 (28.6\%) of the hilar ICCs and in $7(50 \%)$ of the peripheral ICCs. No difference was observed in the expression of arginase-1 between peripheral ICCs showing the major histology of poorly differentiated adenocarcinoma and those showing the major histology of well- or moderately differentiated tubular adenocarcinoma or papillary adenocarcinoma (Table III). All of the hilar ICCs expressing arginase-1 (8 of the 14 hilar ICCs) showed major histology of well- or moderately differentiated tubular adenocarcinoma or papillary adenocarcinoma (Table I).

The neural cell adhesion molecule (N-CAM) has been reported to be a possible marker of hepatic progenitor cells which are assumed to reside in the bile ductules and canals of Hering $(5,14,15)$. Therefore, the expression of N-CAM was examined in peripheral ICCs. N-CAM was expressed in 3 of the 14 peripheral ICCs (Table I, Fig. 6). However, the expression of N-CAM was not associated with the expression of arginase-1 (Table IV).

\section{Discussion}

Immunostaining of grade 5 for CK-19 and CK-7 was observed in $13(92.9 \%)$ of the 14 hilar PI ICCs, and in $7(50 \%)$ of the 14 peripheral MF ICCs. Sasaki et al (16) have reported that 22.2 and $22.2 \%$ of MF ICCs did not reveal immunostaining for CK-19 and CK-7, respectively, in more than $10 \%$ of cancer 


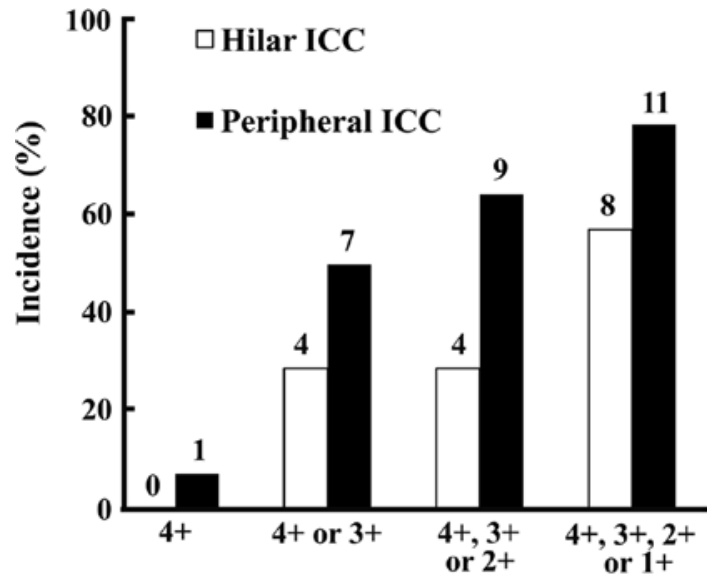

Immunostaining grade

Figure 4. Grades of immunostaining for arginase-1 in the 14 hilar and 14 peripheral ICCs. The immunostaining grades were: $1+, 1 \% \leq \mathrm{p}$ (positive cells) $<5 \% ; 2+, 5 \% \leq \mathrm{p}<10 \% ; 3+, 10 \% \leq \mathrm{p}<40 \% ; 4+, 40 \% \leq \mathrm{p}<70 \% ; 5+\mathrm{p} \geq 70 \%$.
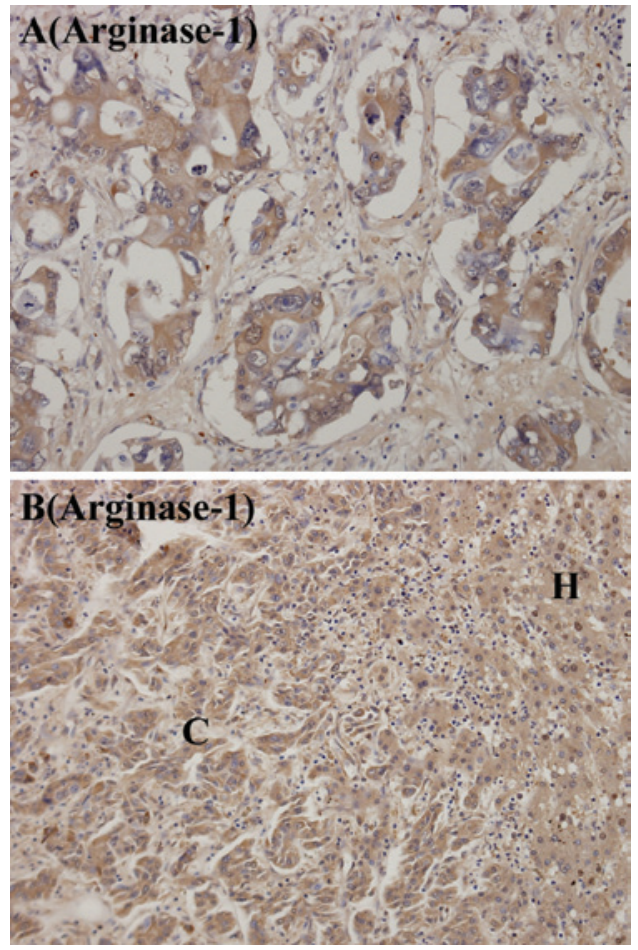

Figure 5. Immunostaining for arginase-1. (A and B) Immunostaining for arginase-1 in areas of moderately differentiated tubular adenocarcinoma and in poorly differentiated adenocarcinoma, respectively. C and $\mathrm{H}$ in (B) show areas of cancer cells and hepatocytes, respectively. Original magnification, $\mathrm{x} 200$.

cells. Aishima et al (17) have reported that 16 and $10 \%$ of MF ICCs did not express CK-19 and CK-7, respectively, in more than $10 \%$ of cancer cells. In addition, D'Errico et al (11) have shown that all of their hilar ICCs and ICCs originating from major bile ducts expressed both CK-19 and CK-7, and all peripheral ICCs expressed CK-19, but $50 \%$ of peripheral ICCs did not express CK-7. Thus, it is likely that at least certain MF ICCs express less CK-19 or CK-7 compared to hilar PI ICCs. This difference may reflect the origin of MF ICCs in

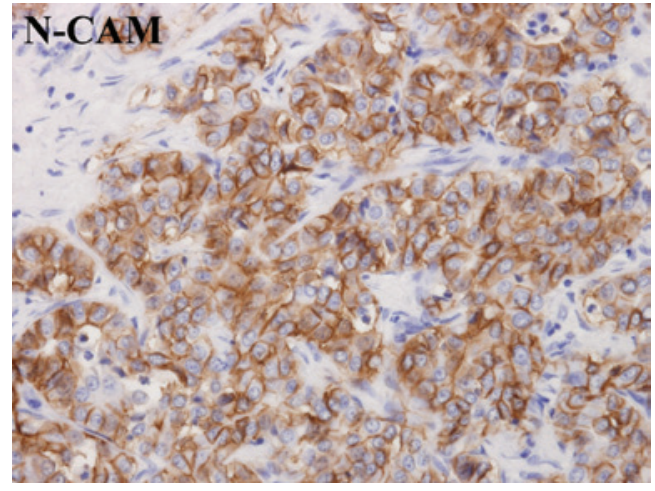

Figure 6. Immunostaining of N-CAM in an area of poorly differentiated adenocarcinoma. Original magnification, $\mathrm{x} 400$.

Table III. Expression of arginase-1 in peripheral intrahepatic cholangiocarcinomas.

\begin{tabular}{lcc}
\hline Grade & $\begin{array}{c}\text { Well- or moderately } \\
\text { differentiated tubular } \\
\text { adenocarcinoma } \\
\text { or papillary } \\
\text { adenocarcinoma } \\
(\mathrm{n}=7)(\%)\end{array}$ & $\begin{array}{c}\text { Poorly differentiated } \\
\text { adenocarcinoma } \\
(\mathrm{n}=7)(\%)\end{array}$ \\
\hline $4+$ & $1(14.3)$ & $0(0)$ \\
$4+$ or $3+$ & $4(57.1)$ & $3(42.9)$ \\
$4+, 3+$ or $2+$ & $5(71.4)$ & $4(57.1)$ \\
$4+, 3+, 2+$ & $6(85.7)$ & $5(71.4)$ \\
or $1+$ & & \\
\hline
\end{tabular}

Immunostaining grades were: $1+, 1 \% \leq \mathrm{p}$ (positive cells) $<5 \% ; 2+$, $5 \% \leq \mathrm{p}<10 \% ; 3+, 10 \% \leq \mathrm{p}<40 \% ; 4+, 40 \% \leq \mathrm{p}<70 \%$.

Table IV. Expression of arginase-1 in N-CAM-positive or negative peripheral intrahepatic cholangiocarcinomas (ICCs).

\begin{tabular}{lc}
\hline Expression of N-CAM & $\begin{array}{c}\text { No. of arginase- } \\
\text { positive ICCs }\end{array}$ \\
\hline Positive $(n=3)(\%)$ & $3(100)$ \\
Negative $(n=11)(\%)$ & $8(72.7)$ \\
\hline
\end{tabular}

the periphery of the intrahepatic biliary tree. In support of this hypothesis, Guedj et al (18) reported a difference in the protein expression profile between hilar and peripheral ICCs.

The expression of HepPar-1 antigen and AFP in ICCs has been reported to be $0-11 \%(8-10,19-21)$ and $0-7 \%(7,19-21)$, respectively. In agreement with these reports, AFP or HepPar-1 antigen was expressed in only $14.3 \%$ of the hilar ICCs and in only $14.3 \%$ of the peripheral ICCs in the present study.

The reason for ICCs expressing hepatocyte markers, such as AFP or HepPar-1 antigen, remains unclear. The double immunostaining for CK-7 and HepPar-1 antigen revealed the presence of cancer cells expressing both CK-7 and HepPar-1 antigen. 
Thus, certain ICCs expressing hepatocyte markers may be derived from hepatic progenitor cells capable of differentiating into cholangiocytes and hepatocytes (14). It is also possible that cancer cells of ICCs originating from cholangiocytes may transdifferentiate into hepatocellular carcinoma (HCC) cells, since mouse gall bladder epithelial cells have been demonstrated to be capable of transdifferentiating into hepatocytes (6).

Recently, Yan et al (12) showed arginase-1 to be a more sensitive hepatocyte marker in HCCs, particularly in poorly differentiated HCCs, compared to HepPar-1 antigen. In that study, HepPar-1 antigen and arginase-1 were positively stained in 46.4 and $85.7 \%$ of poorly differentiated HCCs, respectively. Arginase-1 was shown to be positively stained in only 1 of 6 ICCs, and useful for distinguishing a poorly differentiated HCC from an adenocarcinoma. In the present study, ICCs were stained immunohistochemically for arginase-1 using the same antibody and the same procedure used by Yan et al (12). However, our results show that 4 (28.6\%) of 14 hilar ICCs, and $7(50 \%)$ of 14 peripheral ICCs expressed arginase-1 in more than $10 \%$ of cancer cells. Furthermore, our results demonstrate that $3(42.9 \%)$ of 7 peripheral ICCs, exhibiting a major histology of poorly differentiated adenocarcinoma, expressed arginase- 1 in more than $10 \%$ of cancer cells. These results indicate that care should be taken when using arginase- 1 as a hepatocyte marker for distinguishing a poorly differentiated HCC and a poorly differentiated peripheral ICC.

A ductal reaction, hyperplasia of ductules, observed in the cirrhotic liver, contains N-CAM-positive cells, and intermediate hepatobilliary cells that express hepatocyte and cholangiocyte markers $(14,15)$. Moreover, N-CAM has been reported to be a potential marker of hepatic progenitor cells $(14,15)$. However, in the present study the expression of $\mathrm{N}-\mathrm{CAM}$ was not found to be associated with the expression of arginase-1, suggesting that the expression of arginase-1 in ICCs is independent of its origin.

In conclusion, findings of the present study indicate that the hepatocyte markers, AFP and HepPar-1 antigen, are rarely but definitely expressed in hilar and peripheral ICCs, and that another hepatocyte marker, arginase-1, is expressed at a high frequency in hilar and peripheral ICCs, irrespective of their histology.

\section{Acknowledgements}

The authors thank Mr. K. Kobayashi and Ms. M. Kakihana for their valuable technical support.

\section{References}

1. Sano T, Kamiya J, Nagino M, et al: Macroscopic classification and preoperative diagnosis of intrahepatic cholangiocarcinoma in Japan. J Hepatobiliary Pancreat Surg 6: 101-107, 1999.

2. Yamasaki S: Intrahepatic cholangiocarcinoma: macroscopic type and stage classification.J Hepatobiliary Pancreat Surg 10: 288-291, 2003.
3. Nakanuma Y, Sato Y, Harada K, Sasaki M, Xu J and Ikeda H: Pathological classification of intrahepatic cholangiocarcinoma based on a new concept. World J Hepatol 2: 419-427, 2010.

4. Nakanuma Y, Sasaki M, Ikeda H, et al: Pathology of peripheral intrahepatic cholangiocarcinoma with reference to tumorigenesis. Hepatol Res 38: 325-334, 2008.

5. Roskams T: Liver stem cells and their implication in hepatocellular and cholangiocarcinoma. Oncogene 25: 3818-3822, 2006.

6. Kuver R, Savard CE, Lee SK, Haigh WG and Lee SP: Murine gallbladder epithelial cells can differentiate into hepatocytelike cells in vitro. Am J Physiol Gastrointest Liver Physiol 293: G944-G955, 2007.

7. Maeda T, Kajiyama K, Adachi E, Takenaka K, Sugimachi K and Tsuneyoshi M: The expression of cytokeratins 7, 19, and 20 in primary and metastatic carcinomas of the liver. Mod Pathol 9: 901-909, 1996.

8. Chu PG, Ishizawa S, Wu E and Weiss LM: Hepatocyte antigen as a marker of hepatocellular carcinoma: an immunohistochemical comparison to carcinoembryonic antigen, CD 10, and alpha-fetoprotein. Am J Surg Pathol 26: 978-988, 2002.

9. Fan Z, van de Rijn M, Montgomery K and Rouse RV: Hep Par 1 antibody stain for the differential diagnosis of hepatocellular carcinoma: 676 tumors tested using tissue microarrays and conventional tissue sections. Mod Pathol 16: 137-144, 2003.

10. Mosnier J-F, Kandel C, Cazals-Hatem D, et al: N-cadherin serves as diagnostic biomarker in intrahepatic and perihilar cholangiocarcinomas. Mod Pathol 22: 182-190, 2009.

11. D'Errico A, Baccarini P, Fiorentino M, et al: Histogenesis of primary liver carcinomas: strengths and weaknesses of cytokeratin profile and albumin mRNA detection. Hum Pathol 27: 599-604, 1996

12. Yan BC, Gong C, Song J, et al: Arginase-1: a new immunohistochemical marker of hepatocytes and hepatocellular neoplasms. Am J Surg Pathol 34: 1147-1154, 2010.

13. Nakajima T, Kondo Y, Miyazaki M and Okui K: A histopathologic study of 102 cases of intrahepatic cholangiocarcinoma: histologic classification and modes of spreading. Hum Pathol 19: 1228-1234, 1988.

14. Roskams TA, Theise ND, Balabaud C, et al: Nomenclature of the finer branches of the biliary tree: canals, ductules, and ductular reactions in human livers. Hepatology 39: 1739-1745, 2004.

15. Zhou H, Rogler LE, Teperman L, Morgan G and Rogler CE: Identification of hepatocytic and bile ductular cell lineages and candidate stem cells in bipolar ductular reactions in cirrhotic human liver. Hepatology 45: 716-724, 2007.

16. Sasaki A, Kawano K, Aramaki M, Nakashima K, Yoshida T and Kitano S: Immunohistochemical expression of cytokeratins in intrahepatic cholangiocarcinoma and metastatic adenocarcinoma of the liver. J Surg Oncol 70: 103-108, 1999.

17. Aishima S, Asayama Y, Taguchi K, et al: The utility of keratin 903 as a new prognostic marker in mass-forming-type intrahepatic cholangiocarcinoma. Mod Pathol 15: 1181-1190, 2002.

18. Guedj N, Zhan Q, Perigny M, et al: Comparative protein expression profiles of hilar and peripheral hepatic cholangiocarcinomas. J Hepatol 51: 93-101, 2009.

19. Lau SK, Prakash S, Geller SA and Alsabeh R: Comparative immunohistochemical profile of hepatocellular carcinoma, cholangiocarcinoma, and metastatic adenocarcinoma. Hum Pathol 33: 1175-1181, 2002.

20. Kakar S, Muir T, Murphy LM, Lloyd RV and Burgart LJ: Immunoreactivity of Hep Par 1 in hepatic and extrahepatic tumors and its correlation with albumin in situ hybridization in hepatocellular carcinoma. Am J Clin Pathol 119: 361-366, 2003.

21. Tsuji M, Kashihara T, Terada N and Mori H: An immunohistochemical study of hepatic atypical adenomatous hyperplasia, hepatocellular carcinoma, and cholangiocarcinoma with $\alpha$-fetoprotein, carcinoembrionic antigen, CA19-9, epithelial membrane antigen, and cytokeratins 18 and 19. Pathol Int 49: 310-317, 1999. 